University of Warwick institutional repository: http://go.warwick.ac.uk/wrap

This paper is made available online in accordance with publisher policies. Please scroll down to view the document itself. Please refer to the repository record for this item and our policy information available from the repository home page for further information.

To see the final version of this paper please visit the publisher's website. Access to the published version may require a subscription.

Author(s): B. Dutton, A.R. Clough and R.S. Edwards

Article Title: Near field enhancements from angled surface defects: a comparison of scanning laser source and scanning laser detection techniques

Year of publication: 2011

Link to published article: http://dx.doi.org/10.1007/s10921-011-0091-y

Publisher statement: Dutton, B., et al. (2011). Near field enhancements from angled surface defects ; a comparison of scanning laser source and scanning laser detection techniques. Journal of Nondestructive Evaluation, 30(2), pp. 64-70. The original publication is available at www.springerlink.com 


\title{
Near field enhancements from angled surface defects; a comparison of scanning laser source and scanning laser detection techniques
}

\author{
B. Dutton, A.R. Clough and R.S. Edwards \\ Department of Physics, University of Warwick, Coventry, CV4 7AL, UK \\ b.dutton@warwick.ac.uk
}

\begin{abstract}
Enhancement of the Rayleigh wave signal amplitude at a surface defect, due to interference of incident, reflected and mode converted waves, has been reported by several authors, and it has been suggested that this could be used as a fingerprint of the presence of such cracking. The scanning laser line source technique in particular, where signal amplitude is enhanced as the laser generating the Rayleigh waves is in the region of a surface defect, has been reported as a suitable detection tool. However, the previous work has looked primarily at defects propagating normal to the surface, which may not always be a suitable approximation, and the enhancement measured when a detection laser rather than a generation laser is near a crack may, in some cases, be more significant. This work explores near field effects for both laser generation and laser detection points near a defect, and compares the enhancements for defects which are angled relative to the surface. We use a combination of finite element method models and experimental results, and probe enhancements of both the amplitude and frequency signals, and show that scanning the detection point may be a better method for locating surface defects if they are inclined at an angle to the surface.
\end{abstract}

Keywords: Rayleigh wave, enhancement, surface defect, NDT, ultrasound 


\section{Introduction}

Surface crack detection and characterisation are very important in industry; cracks in materials can lead to failure, and it is important to both detect and characterise cracking before it grows deep into the material. Ultrasound is in common use, as testing can be performed in a non-destructive manner, and recent advances in understanding the interaction of surface waves, such as Rayleigh waves, with surface cracking is leading to new techniques for characterisation [1-7]. Recent work has investigated the reflection and transmission of waves following interaction with surface defects which are oriented normal to the sample surface, a geometry which is typical of calibration samples [1,5-8]. It has been seen that a surface defect will act like a filter to an incident wave, hence the transmitted signal for a broadband Rayleigh wave, typical of that generated using non-contact techniques such as electromagnetic acoustic transducers (EMATs) or laser ultrasound, drops off approximately exponentially with depth $[9,10]$.

Enhancement of the ultrasonic signal in the near-field of a defect has been reported $[2,11,12]$, again only for defects oriented normal to the surface. For a detection point close to a defect, interference between the incident Rayleigh wave with the reflected Rayleigh wave, plus the mode-converted surface skimming longitudinal wave, leads to constructive interference a short distance before the defect, and a significantly larger signal than the incident wave amplitude away from the defect [2,11]. This has been modelled and experimentally confirmed using EMATs [11].

When using laser generation of ultrasound, the shape of the source and the boundary conditions play a large role in determining the amplitude and frequency content of a signal [13]. If the laser generation point is over a defect, part of the beam is truncated and the boundary conditions change significantly, and research has shown that this leads to a similar 
signal amplitude enhancement during scanning of a sample [2,5-7,12,14]. This enhancement has been the subject of much recent research $[2,5-7,12,14]$ and is known as the scanning laser source (SLS) technique, or scanning laser line source (SLLS) if the generation laser is focussed into a line. Frequency enhancements have also been reported before, with researchers investigating changes in the frequency at which the fast Fourier transform (FFT) shows a maximum magnitude [2]. This technique has been suggested as a useful method for fingerprinting the position of a defect, and measurements of partially closed defects show a non-linear frequency enhancement [12].

It is essential to understand fully how ultrasound interacts with surface cracks, in order to gain an optimal characterisation of the crack geometry. The work highlighted above has used primarily simulated defects, such as machined slots, which have been machined normal to the sample surface. This will not necessarily be a suitable approximation for all defects, for example rolling contact fatigue in rails which grows at an angle to the surface, or branched defects such as stress corrosion cracking. It is therefore important to understand if there are differences between the Rayleigh wave interaction with defects normal to the surface and those at an angle to the surface normal. There has been limited work on this; Kinra et al. performed initial investigations of the interaction of Rayleigh waves with angled defects [15]. Several researchers in diverse areas including geophysics have studied the interaction of a Rayleigh wave incident on a wedge [16,17], but have not considered a finite depth defect. We have recently studied the changes in reflection and transmission coefficients with angle for several different defect depths, using a combination of models and experiments using laser generation and detection of ultrasound [18-20]. These results show an angle dependence both in the reflection and transmission of Rayleigh waves, and furthermore a variation with angle in the arrival time for various bulk wave modes generated by mode conversion of the incident 
Rayleigh wave at the crack $[18,20]$, and these effects could be exploited for identifying the angle of the defect.

During this research, interesting effects in the signal and frequency enhancement in the near-field have been observed. This paper reports these effects, considering both amplitude and frequency enhancement behaviour during scanning across cracks which have different angles to the surface. We compare the effect of scanning the laser line source (SLLS) or the detector (SLD, scanned laser detector) across the defect, and show that scanning the detector may have significant advantages over a scanned generation point if defects are angled, rather than normal to the surface. We also investigate frequency enhancements with angle, to highlight further potential measurement techniques when using a scanning detection system and the benefits over scanning the generation point.

\section{Model and experimental details}

This paper considers laser generation and detection of ultrasound. Laser generation will typically generate a broadband Rayleigh wave, and can be used in either the thermoelastic regime (causing no damage to the surface) or the ablative regime, where the surface of the sample suffers some damage [21]. For detection, we consider laser detection using a twowave mixer interferometer system, which is sensitive to the out-of-plane (OP) component of the Rayleigh wave. Both experiments and models are used, and Figure 1 shows the measurement configuration and the sample geometry, including the surface defect.

To explore near field amplitude and frequency enhancement behaviours as crack angle varies, two different 3D finite element method (FEM) models were generated with a software package, PZFlex [22].

2.1 Scanning laser detection 
The SLD model was generated using loading forces derived from a laser pulse duration of 10 ns to give a good comparison with the experimental laser pulse used; these forces were applied onto the sample in the form of dipoles $[14,23]$. The model configuration was set up as shown in Figure 1, with results taken for different detection positions to simulate a scan. Samples had defects with angles ranging from $10^{\circ}$ to $170^{\circ}$ and a normalised length of $\mathrm{d} / \lambda=1.11$, with other depths investigated and reported in the studies of transmission of the Rayleigh wave [18-20]. The sample top surface and the crack faces of both models were assigned to be 'Free'. All other surfaces were assigned to be 'Absorbing' to simulate a larger sample, saving computational memory and processing time. Symmetry was also applied to improve model efficiency.

\subsection{Scanning laser line source}

For the SLLS technique, consideration must be made of the changes in boundary conditions and source truncation near a defect, which leads to complications when using a dipole force model. In this case, the heat-mechanical bridge mode in PZFlex was used, with the crack walls free to expand [22]. For this model the laser line source spatial and temporal temperature profile, based on the experimental line source $[12,23]$, were used, with the heat model creating a material expansion which in turn generated ultrasound waves in the material. For SLLS the detection and generation positions shown in Figure 1 were exchanged, therefore it is the generation line source that is scanned across the crack surface. These models had defect angles, $\theta$, ranging from $10^{\circ}$ to $170^{\circ}$ and a normalised length of $\mathrm{d} / \lambda=1.11$, with a limited number of models produced when compared to the SLD model due to much longer processing times.

\subsection{Experimental scans}


To validate the models, experimental scans were performed on aluminium samples with dimensions 150x150x50mm using non-contact laser-ultrasound to both generate and detect, and the near field enhancement behaviour was investigated. To generate the surface waves in a thermoelastic manner a Nd:YAG laser with 10 ns rise time was used. The laser beam was focused into a line with dimensions $6 \mathrm{~mm}$ by $500 \mu \mathrm{m}$, which generated ultrasound in a direction perpendicular to the laser line; this method also has the effect of increasing the frequency bandwidth of the generated surface waves [24,25]. Detection of the ultrasound waves was performed using a two-wave mixer from IOS, which is capable of measuring the out-of-plane surface displacement without the need for surface preparation such as polishing [26]. Unpolished fabricated aluminium samples possessed crack angles, $\theta$, from $30^{\circ}$ to $150^{\circ}$, with a normalised length of $d / \lambda=1.11$.

\section{Results: amplitude enhancements}

The signal enhancements reported previously and described above, observed as a scan is performed across a crack, have been reported for cracks which are normal to the surface only $[2,11,12]$. One paper [11] reports the detection point passing over a crack, whereas the SLS technique with the laser source scanned over the crack has received much recent attention $[2,5-7,12,14]$. The crack angle dependence of the enhancement has previously been ignored. Here we report measurements of this behaviour as the crack angle varies, comparing both SLD and SLLS techniques.

\subsection{Amplitude enhancement for scanned laser detection}

The first scanning technique presented is SLD. As the detection point passes close to the defect, constructive interference between the incident and reflected Rayleigh waves and the mode converted surface skimming longitudinal, lead to the enhancement for a $90^{\circ}$ defect [11]. For these experiments, as the detection point was scanned across the surface crack, 
changes in the amplitude of the windowed Rayleigh wave were monitored. A typical B-scan from modelled results is shown in Figure 2(a) for a $40^{\circ}$ crack (angle defined in Figure 1), where the out-of-plane amplitude of the signals is shown by the grey-scale. The windowed Rayleigh wave is shown by the dashed lines. Within this time window, the maximum peakto-peak amplitude was measured and is plotted in Figure 2(b). The defect was at a scan position of $45.5 \mathrm{~mm}$.

To calculate the enhancement of the signal, firstly the amplitudes before the crack were considered. Attenuation of the Rayleigh wave as the separation between generation and detection points is small over these distances; however, it was taken into account when calculating the no-defect signal amplitude at the enhancement position. The enhancement factor was then calculated as the ratio of the amplitude of the enhanced signal to that which would have been measured without a defect. For this particular orientation of crack $\left(40^{\circ}\right)$ the signal was enhanced by a factor of 10.8 above the signal amplitude when no defect is present.

This amplitude enhancement calculation was performed for each crack angle using the model data, and is shown in Figure 3 for both out-of-plane (OP, open squares) and in-plane (IP, open circles) displacements. In addition, the out-of-plane experimental data for several crack angles between $30^{\circ}$ and $150^{\circ}$ are also displayed on the same figure as solid squares and show a very good agreement with the model data. Experimental data is limited to out-ofplane displacements only due to the interferometer technique used, however, the use of EMATs allows measurement of the velocity components in both the out-of-plane and inplane, and will be the subject of a later publication. The reduced experimental enhancement at some angles is mainly due to focussing issues, leading to a larger detection point size when compared with the model [11]. Nevertheless, the out-of-plane enhancement for this depth of defect for both model and experimental data show an approximately exponential dependence 
on crack angle, shown as a solid line in Figure 3 . The data point at $10^{\circ}$ was not included in this fit; at this angle the vertical depth of the defect is very small compared to the wavelength, and the majority of the waves are able to penetrate beneath the defect $[9,27]$.

The modelled and experimental enhancements for a $90^{\circ}$ crack agree well with previously published work [11], and each of the enhancements measured (out-of-plane and in-plane for the model, out-of-plane for the experiments) exhibits an general trend of increasing enhancement as the crack angle decreases, to a maximum at a certain angle. This enhancement is known to be an excellent fingerprint of the defect, and clearly becomes more so as the angle decreases.

The enhancement for angles other than $90^{\circ}$ is again due to constructive interference of Rayleigh and mode-converted waves close to the defect, with the reflected Rayleigh and mode-converted surface skimming longitudinal wave dominating for angles around $90^{\circ}$ $[1,11]$. The exact mechanism for the enhancement at lower angles warrants further investigation; we must consider the effect of the waves which have been mode-converted from Rayleigh waves at the crack tip and have arrival times close to those of the incident Rayleigh wave, and constructive interference between incident and reflected Rayleigh waves and these mode-converted waves. It is clear from a comparison of the in-plane and out-ofplane enhancements, and the difference in their behaviour, that these mode-converted waves play an extremely important role [28].

\subsection{Amplitude enhancement for scanned laser generation}

We now compare these enhancements with the SLLS method, which has received much recent attention $[2,5-7,12,14]$. In this case, the laser source passes over the crack, generating an incident Rayleigh wave which can be reflected and mode-converted at the defect. However, attenuation of the mode-converted surface skimming longitudinal wave means that 
by the time the signals reach the detection point its effect will be minimal. Enhancement for the SLLS method therefore considers interference of the incident and reflected Rayleigh waves, but also the changes in the laser generation shape and boundary conditions as the laser passes over the crack $[2,5-7,12,13]$.

Similarly to the analysis of the SLD data, the Rayleigh wave peak-to-peak amplitude was monitored during a scan and the ratio of the enhanced signal to the incident amplitude calculated for each crack angle. Figure 4 shows the out-of-plane amplitude enhancement calculated from the experimental data for crack angles of $30^{\circ}, 45^{\circ}, 90^{\circ}, 145^{\circ}$ and $150^{\circ}$, and both the out-of-plane and in-plane enhancements calculated from the modelled data. Due to time limitations running the SLLS model, results from fewer crack angles than for SLD are displayed, however, results again show very good agreement between modelled and experimental data. In this case, the out-of-plane and in-plane enhancements from the model data show a very similar variation with angle, with a much less significant angle-dependence to the enhancements than measured when using a scanned detection system.

The fit to the out-of-plane enhancement from the scanned laser detection measurements is shown in Figure 4 as a solid line. This shows clearly that the measured enhancement as the angle reduced is much larger when the detection point is scanned over the crack than when scanning the laser generation point over the defect. It is clear that for SLLS, the main contribution to the enhancement is the change in the generation conditions, which will affect both the in-plane and out-of-plane components in a similar manner, and lead to much less of an angle dependence.

\section{Results: frequency enhancements}

Previous measurements have looked at the effect of a surface defect on the frequency content of a signal, for example by measuring changes in transmitted frequency content [9]. With the 
SLLS technique, the frequency content in the region of the enhancement has been considered. Kromine et al. showed that the frequency at which the FFT had a maximum magnitude showed some variation in the region of a defect, changing by a measurable amount [2]. Other measurements have shown interesting non-linear effects when measuring partially closed cracks, and have shown that enhancement at higher frequencies may be beneficial when detecting surface defects [12]. Here we consider the changes in frequency content with angle when scanning using SLD and SLLS.

\subsection{Frequency enhancement for scanned laser detection}

For each position of a scan, the out-of-plane Rayleigh wave displacement (shown in the BScan in Figure 2) was windowed and an FFT performed. These FFTs were then stacked into a frequency B-Scan in a similar manner to producing B-Scans from time-domain data [12], with examples shown in Figure 5(a) and (d) for modelled data with $d / \lambda=1.11$, for a $40^{\circ}$ and a $90^{\circ}$ crack. The crack is at a position of $45.5 \mathrm{~mm}$ on the scan. Enhancement of the signal is clear near the defect (shown as a dotted line), and a measurement of the maximum magnitude of the FFT at each position would yield very similar results to the amplitude enhancement measurements. However, there are also other interesting frequency effects which require investigation.

Figures 5(a) and (d) show a near-constant frequency content when the generation and detection points are away from the crack, as expected, but the enhancement is clear in the larger magnitudes (shown by the colour scale) close to the defect. The bands near the defect at higher frequencies are due to windowing effects, whereby the separation between the incident and reflected Rayleigh waves leads to a second frequency content within the time window. It is clear, however, that there are certain differences between the two frequency Bscans; at $40^{\circ}$, Figure 5(a), there is a large enhancement at higher frequencies, whereas for $90^{\circ}$ 
this higher frequency enhancement is less significant. This is shown more clearly in Figure 5(b), where the FFTs are shown for generation and detection away from a defect (dotted line), and for the detection point close to a $40^{\circ}$ (solid black line) or $90^{\circ}$ (dashed line) defect. These have been normalised to the peak value of the FFT taken away from a defect.

From Figure 5(b), it is clear that in the region of frequency content of the incident Rayleigh wave (around 0.2-1.2 MHz) the magnitude enhancement on comparing $90^{\circ}$ and $40^{\circ}$ defects follows a similar pattern to that for the signal amplitude, with a higher enhancement for shallower angles. There also appears to be enhancement to higher frequencies for the shallower defect, although variations in this frequency content are overshadowed by windowing effects and an alternative frequency analysis tool may be beneficial here. Figure 5(c) shows similar results in the experimental measurements, where again the enhancement is much larger for the $40^{\circ}$ crack and persists to higher frequencies; the frequency content here is higher than for the models, however the defect depth was scaled to the central wavelength in the ultrasound pulse.

This frequency-dependent behaviour is, to some extent, expected, considering the filtering action of a crack. As shown in reference [9], when a broadband Rayleigh wave is incident on a surface-breaking defect the higher frequencies tend to be blocked (reflected), while the lower frequencies tend to be transmitted. When considering enhancement due to only interaction of incident and reflected Rayleigh waves it is therefore to be expected that the enhanced signal will contain significant higher frequency content. The addition of modeconverted waves will complicate matters, but these are also likely to tend towards higher frequency content [1].

Both model and experimental data confirm that the enhancement in the frequency content is also an excellent fingerprint of the presence of an angled defect. As shown for partially 
closed defects, a measure of the signal at a higher frequency than is generally present in the generated pulse may give a very clear indication of the defect [12].

\subsection{Frequency enhancement for scanned laser line source}

Finally, to compare the two techniques, FFTs were produced for the scanned laser line source for two cases; $45^{\circ}$ and $90^{\circ}$ crack angles, for both model and experimental data. These are shown in Figure 6(a) (model) and (b) (experiment). The SLLS model and experimental data have similar bandwidth and the Rayleigh central frequencies are close, with experimental peak frequency shifts due to focussing issues. Similarly to the amplitude enhancement technique, Figures 6(a) and (b) show larger enhancements for shallower angles, but the differences are much less significant than those found using a scanned detector. In contrast to the SLD measurements, Figure 6 shows no significant frequency enhancement at higher frequencies.

\section{Conclusion}

Previous work has shown that the Rayleigh wave signal enhancement for a surface defect oriented normal to the sample surface, when using either scanned detection or a scanned laser line source to generate signals, can be an excellent fingerprint of the position of a defect. We have shown here that the enhancements in both the amplitude and frequency content, and for both techniques, show promise for use in identifying surface defects and in finding their orientation to the surface and position.

The out-of-plane amplitude enhancements have been compared with those from measurements, where laser detection techniques were used to measure the out-of-plane surface displacement on the sample surface during a scan, and show very good agreement. For SLD measurements both model and experiment show an approximately exponential 
dependence on crack angle, apart from for very shallow angles where the wave is able to pass underneath the defect due to the small vertical depth. From the modelled data, the in-plane and out-of-plane enhancements showed a difference in size, with a cross-over at angles below $40^{\circ}$. This change in behaviour is due to the mode-converted wavemodes present close to the defect, which interfere with the incident and reflected Rayleigh waves [1].

For SLLS measurements some angle dependence is seen, however, it is not as pronounced as that for the SLD measurements. For this case the OP and IP components show a similar dependence on angle, confirming that the majority of the enhancement comes from changing generation conditions, as expected.

Measurements using SLD techniques showed large frequency enhancements for shallow cracks, and exhibited enhancement at higher frequencies than were present prior to enhancement. For the SLLS measurements, some angle dependence to the frequency enhancement was observed, but it was much less significant than for SLD measurements.

This angle dependence of both the amplitude and frequency content could potentially be used to identify different angled cracks in real samples. It is clear for these measurements that for defects which are inclined at an angle to the surface, such as rolling contact fatigue in rails, which initially propagates at an angle of around $25^{\circ}$ to the surface, SLD may be the more beneficial technique for identifying and characterising defects when compared with SLLS. It must be remembered that a defect with an angle of greater than $90^{\circ}$ to the propagation direction of the Rayleigh wave will have an angle of less than $90^{\circ}$ if the generation and detection points are switched, and hence the large enhancements at shallow angles can again be exploited.

\section{Acknowledgements}


This work was funded by the European Research Council under grant 202735, ERC Starting Independent Researcher Grant.

\section{References}

1. Jian, X., Dixon, S., Guo, N., and Edwards, R.: Rayleigh wave interaction with surfacebreaking cracks. Journal of Applied Physics 101 (6), 064906 (2007)

2. Kromine, A.K., Fomitchov, P.A., Krishnaswamy, S. and Achenbach, J.D.: Laser ultrasonic detection of surface breaking discontinuities: Scanning laser source technique. Materials Evaluation 58 (2), 173 (2000)

3. Fan, Y., Dixon, S., Edwards, R.S. and Jian, X.: Ultrasonic surface wave propagation and interaction with surface defects on rail track head. NDT \& E International 40 (6), 471 (2007)

4. Edwards, R.S., Jian, X., Fan, Y. and Dixon, S.: Rail defect detection using ultrasonic surface waves. Review of Progress in Quantitative Nondestructive Evaluation, Vols 25A and 25B, in AIP Conference Proceedings 820, pp. 1601-1608 (2005)

5. Jeong, H. and Park, M.C.: Finite-element analysis of laser-generated ultrasounds for wave propagation and interaction with surface-breaking cracks. Research in Nondestructive Evaluation 16 (1), 1 (2005)

6. Sohn, Y. and Krishnaswamy, S.: Interaction of a scanning laser-generated ultrasonic line source with a surface-breaking flaw. Journal of the Acoustical Society of America 115 (1), $172(2004)$

7. Sohn, Y. and Krishnaswamy, S.: A near-field scanning laser source technique and a microcantilever ultrasound receiver for detection of surface-breaking defects. Measurement Science and Technology 17 (4), 809 (2006)

8. Vu, B.Q. and Kinra, V.K.: Diffraction of Rayleigh-waves in a half-space 1. Normal edge crack. Journal of the Acoustical Society of America 77 (4), 1425-1430 (1985) 
9. Edwards, R.S., Dixon, S. and Jian, X.: Depth gauging of defects using low frequency wideband Rayleigh waves. Ultrasonics 44 (1), 93 (2006)

10. Wang, J.S., Xu, X.D., Liu, X.J. and Xu, G.C.: A tuneable acoustic filter made by periodical structured materials. Applied Physics Letters 94 (18), 181908 (2009)

11. Edwards, R.S., Jian, X., Fan, Y. and Dixon, S.: Signal enhancement of the in-plane and out-of-plane Rayleigh wave components. Applied Physics Letters 87 (19), 194104 (2005)

12. Dixon, S., Cann, B., Carroll, D. L., Fan, Y. and Edwards, R. S.: Non-linear enhancement of laser generated ultrasonic Rayleigh waves by cracks. Nondestructive Testing and Evaluation 23 (1), 25 (2008)

13. Scruby, C.B. and Drain, L.E., Laser Ultrasonics: Techniques and Applications. Bristol: Adam Hilger, (1990)

14. Arias, I. and Achenbach, J.D.: A model for the ultrasonic detection of surface-breaking cracks by the scanning laser source technique. Wave Motion, 39 (1), 61 (2004)

15. Kinra, V.K.: Diffraction of Rayleigh-waves in a half-space 2. Inclined edge crack. Journal of the Acoustical Society of America 79 (6), 1688 (1986)

16. Budaev, B.V. and Bogy, D.B.: Rayleigh-wave scattering by a wedge. Wave Motion 22 (3), 239 (1995)

17. Fujii, K.: Rayleigh-wave scattering at various wedge corners - investigation in the wider range of wedge angles. Bulletin of the Seismological Society of America 84 (6), 1916 (1994)

18. Dutton, B., Rosli, M.H. and Edwards, R.S.: Defect feature extraction using surface wave interactions and time-frequency behaviour. Review of Progress in Quantitative Nondestructive Evaluation, Vols 29A and 29B, in AIP Conference Proceedings 1211, pp. $647-654(2010)$ 
19. Dutton, B., Clough, A.R., Rosli, M.H. and Edwards, R.S.: Exploring surface wave interaction with angled defects in the near and far field. Journal of Physics: Conference Series, 2nd International Symposium of Laser-Ultrasonics, in press.

20. Dutton, B., Clough, A.R., Rosli, M.H. and Edwards, R.S.: Non-contact ultrasonic detection of angled surface defects. NDT \& E International, in press.

21. Essex, S.D., Potter, M. D. G., Vann, R. and Dixon, S.: Utilizing EBSD to validate and understand NDE techniques. Review of Progress in Quantitative Nondestructive Evaluation, Vols 28A and 28B, in AIP Conference Proceedings 1096, pp. 1224-1231 (2009)

22. PZFlex version 2.3, developed by Weidlinger Associates, Inc., http://www.pzflex.com/

23. Arias, I. and Achenbach, J.D.: Thermoelastic generation of ultrasound by line-focused laser irradiation. International Journal of Solids and Structures 40 (25), 6917 (2003)

24. Aindow, A.M., Dewhurst, R.J. and Palmer, S.B.: Laser-Generation of Directional Surface Acoustic-Wave Pulses in Metals. Optics Communications 42 (2), 116 (1982)

25. Hutchins, D.A.: Mechanisms of Pulsed Photoacoustic Generation. Canadian Journal of Physics 64 (9), 1247 (1986)

26. Klein, M.B., Bacher, G. D., Grunnet-Jepsen, A., Wright, D. and Moerner, W. E.: Homodyne detection of ultrasonic surface displacements using two-wave mixing in photorefractive polymers. Optics Communications 162 (1-3), 79 (1999)

27. Viktorov, I.A.: Rayleigh and Lamb waves: physical theory and applications. Ultrasonic technology, New York: Plenum Press (1967)

28. Dutton, B., Clough, A.R. and Edwards, R.S.: Ultrasound near field enhancement mechanism for angled surface defects. In preparation 
Figure list

Fig. 1 Model and experimental sample scanning details. The scanning detection point case is shown, and for SLLS the source and detection were reversed. All dimensions in $\mathrm{mm}$.

Fig. 2 Results from a modelled scanned detection out-of-plane measurement of a $40^{\circ}$ crack. (a) is the B-Scan with dashed window around the Rayleigh-wave arrival time; (b) is the peakto-peak Rayleigh wave amplitude vs. scan position.

Fig. 3 Scanned laser detection: out-of plane and in-plane amplitude enhancements vs. crack angle, for $\mathrm{d} / \lambda=1.11$. The horizontal line shows no enhancement (factor=1).

Fig. 4 Scanned laser line source: signal enhancement factor vs. crack angle for out-of-plane and in-plane modelled data and out-of-plane experimental data. The fit to the out-of-plane SLD data is shown as a solid line. All data is for $\mathrm{d} / \lambda=1.11$.

Fig. 5 Magnitude FFT B-scan of modelled out-of-plane displacement data using a scanned laser detector for (a) $40^{\circ}$ crack and (d) $90^{\circ}$ crack, and the corresponding normalised FFTs (b). (c) shows experimental normalised FFTs for $40^{\circ}$ and $90^{\circ}$ cracks.

Fig. 6 Out-of-plane SLLS normalised FFTs for $45^{\circ}$ and $90^{\circ}$ cracks from, (a) model and (b) experimental. 


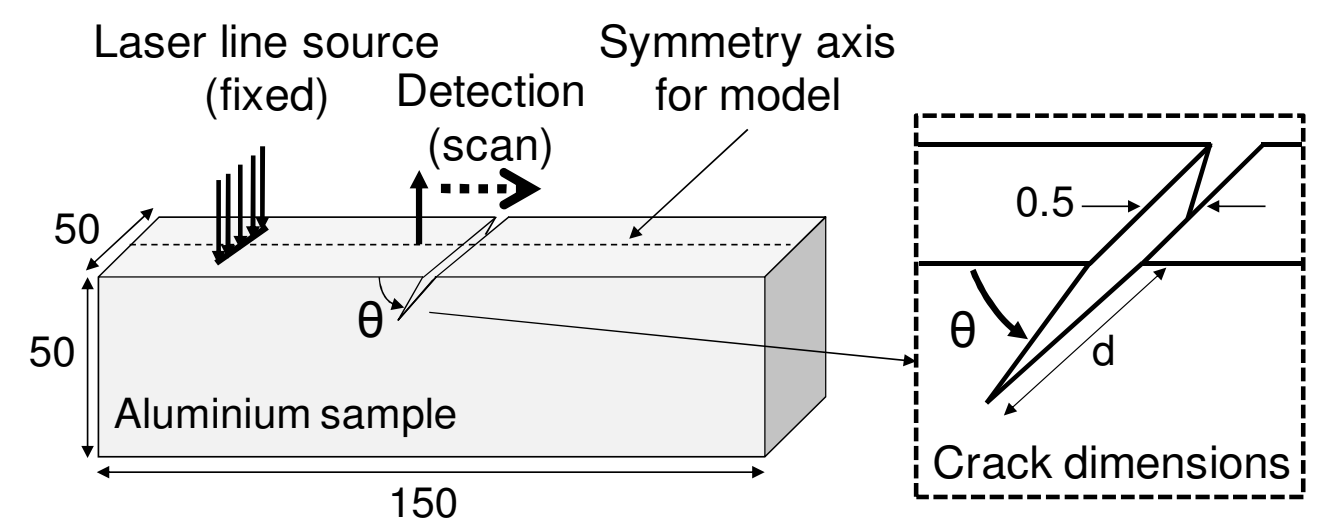

Fig. 1
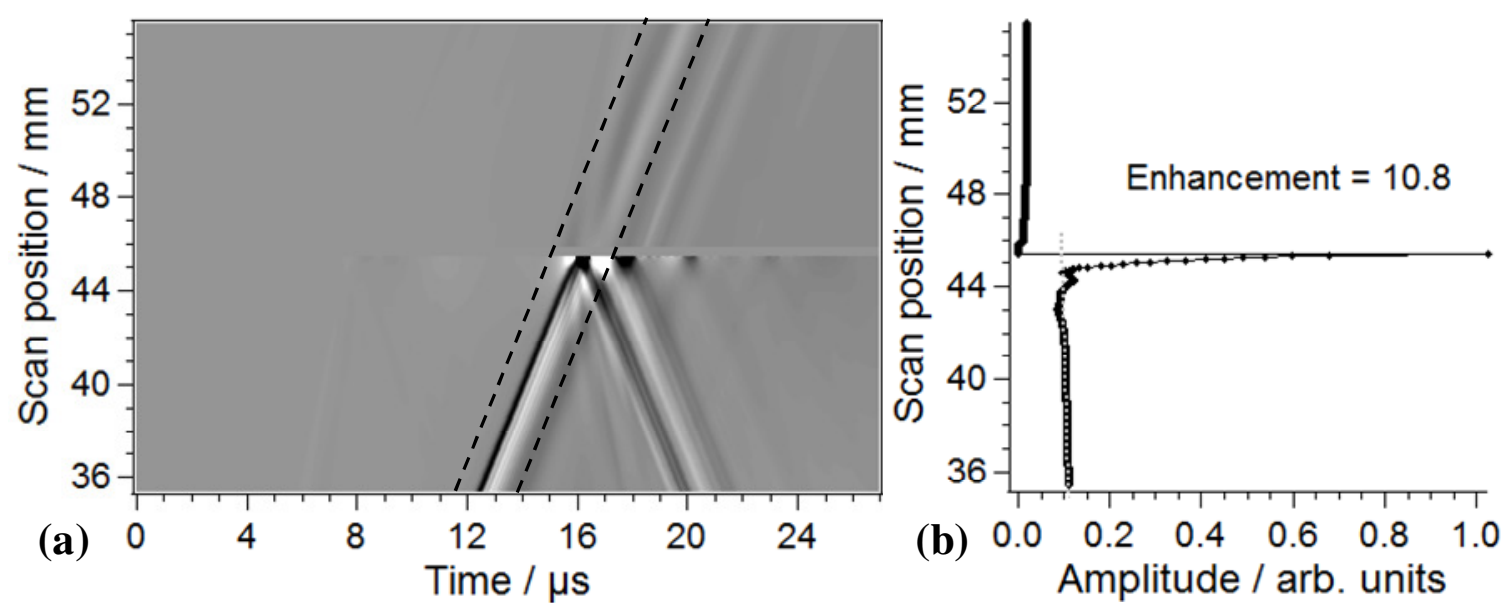

Fig. 2

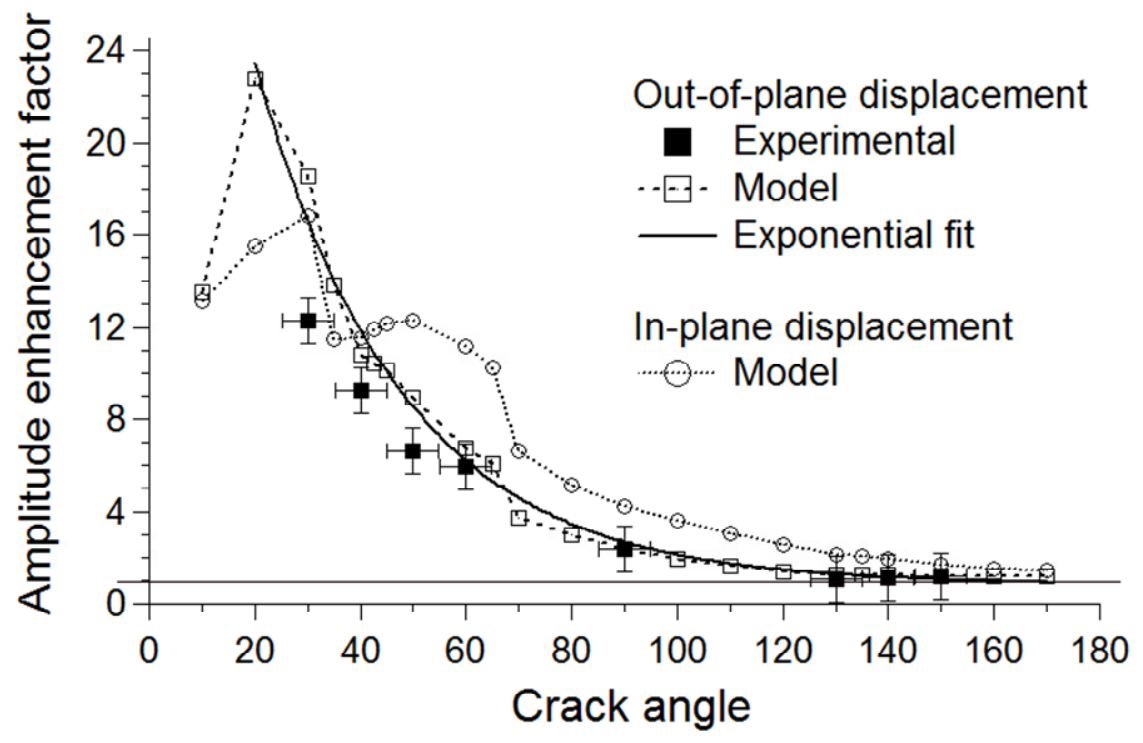

Fig. 3 


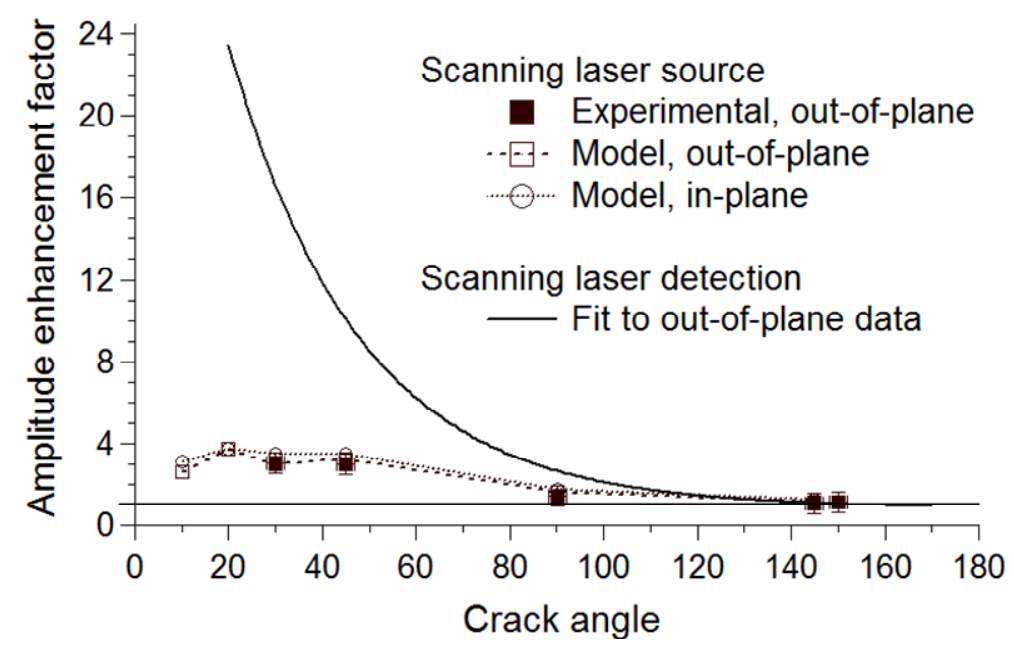

Fig. 4

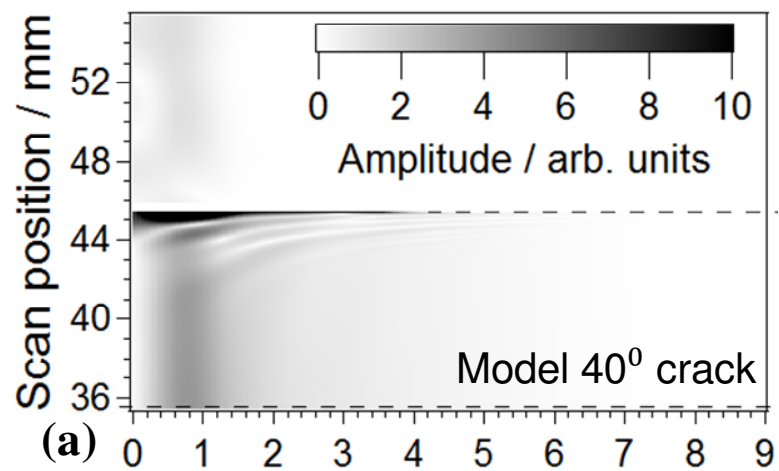

Frequency / MHz
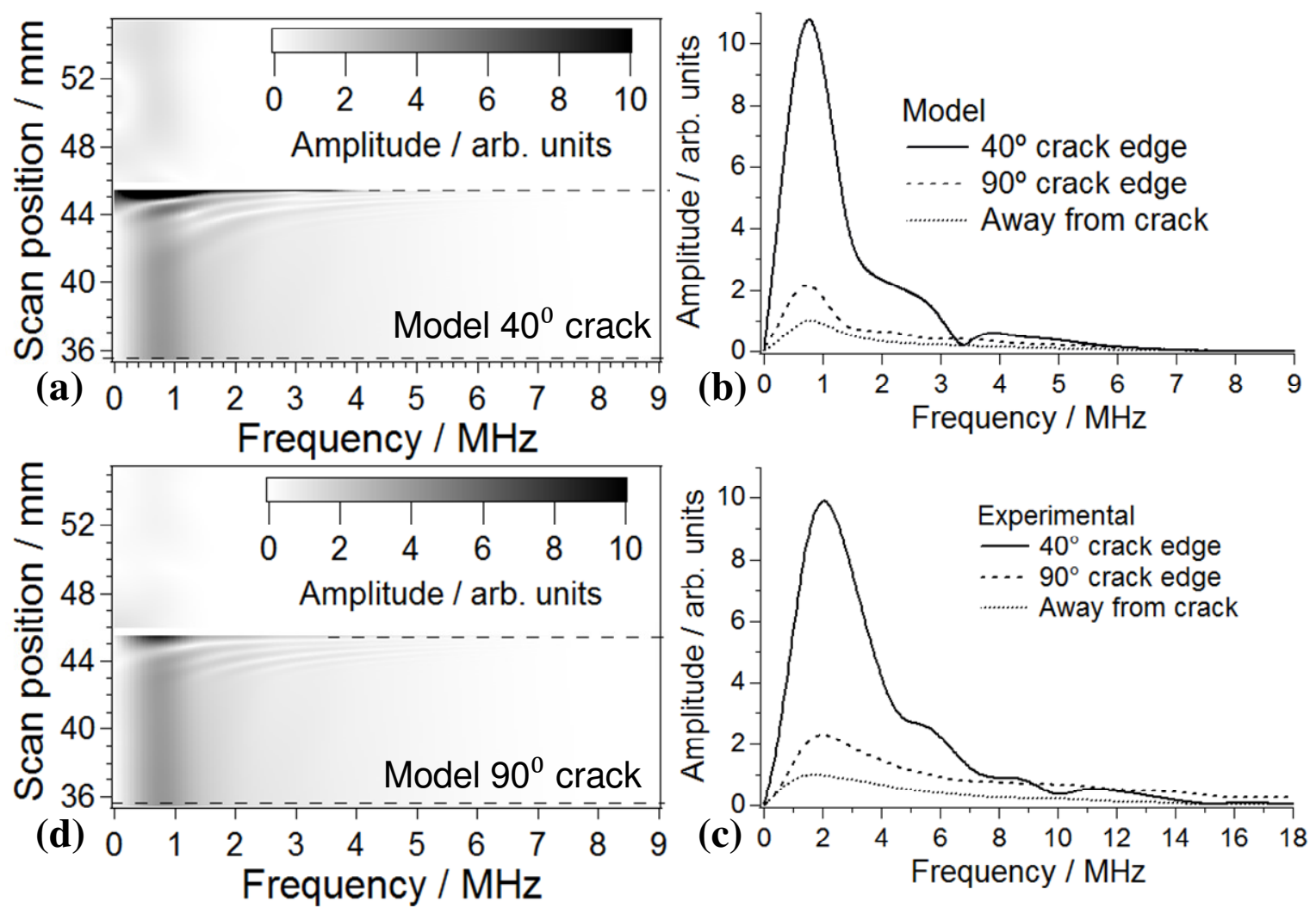

Fig. 5 

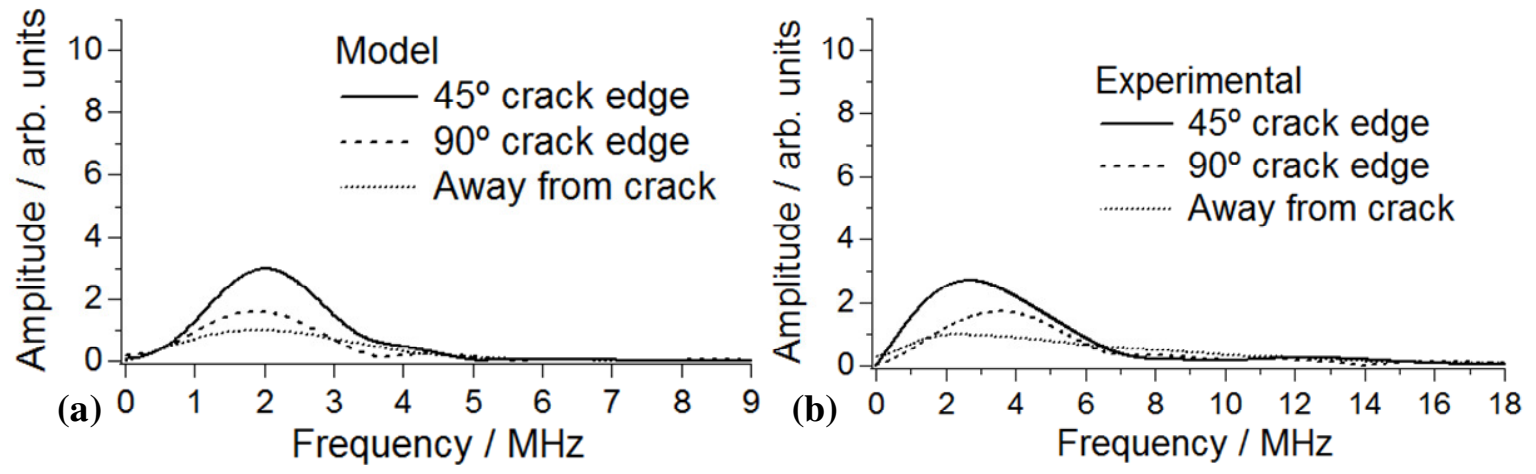

Fig. 6 